le portiQue $\begin{array}{ll}\text { Le Portique } \\ \text { Revue de philosophie et de sciences humaines }\end{array}$

$29 \mid 2012$

Georges Bataille

\title{
La parodie dans tous ses états
}

Parody, every which way

Über die Parodie

\section{François Warin}

\section{OpenEdition}

Journals

Édition électronique

URL : http://journals.openedition.org/leportique/2601

DOI : $10.4000 /$ leportique.2601

ISSN : $1777-5280$

Éditeur

Association "Les Amis du Portique"

Édition imprimée

Date de publication : 25 octobre 2012

ISSN : 1283-8594

\section{Référence électronique}

François Warin, "La parodie dans tous ses états », Le Portique [En ligne], 29 | 2012, document 5, mis en ligne le 15 décembre 2014, consulté le 26 mars 2021. URL : http://journals.openedition.org/ leportique/2601; DOI : https://doi.org/10.4000/leportique.2601

Ce document a été généré automatiquement le 26 mars 2021.

Tous droits réservés 


\title{
La parodie dans tous ses états
}

\author{
Parody, every which way \\ Über die Parodie
}

François Warin

Siamo contenti?

son dio, ho fatto questa caricatura.

F. Nietzsche

1 Quand je songe à Bataille je vois d'abord ce portrait de lui où son regard infiniment doux et légèrement extasié semble ouvert à ce qu'il appelait le bleu du ciel : ouvert sur l'essence sans limites de l'être impossible, ouvert sur ce qu'il nommait le il y a, il y a qui ne pouvait appeler qu'un non-savoir: nous ne pouvons égaler ce ciel vide qui nous traite infiniment en meurtrier, écrivait-il, nous anéantissant jusqu'au dernier.

2 Et si je reviens aujourd'hui sur celui qui a continué à hanter la plupart des textes que j'ai pu écrire ${ }^{1}$ c'est pour m'interroger à nouveau sur ce Nietzsche et Bataille qui en a inauguré la série, quitte à réécrire et à faire, pour rien peut-être, et vingt ans après, un nouveau tour de manège. Da capo ? Si un tel titre semblait céder à un stéréotype, celui de l'exercice très académique de la comparaison, il cherchait en vérité plus secrètement à répondre - son sous-titre, (la parodie à l'infini) l'indique - à une difficulté : comment écrire sur Bataille, comment surmonter cette aporie qu'il rencontra lui même lorsqu'il écrivit sur Nietzsche, puisque comme il le disait: nul ne peut lire Nietzsche sans être Nietzsche : je ne pouvais qu'écrire avec ma vie le livre projeté sur Nietzsche ${ }^{2}$.

D'une certaine façon, pourtant, rien n'est plus simple que d'exposer la pensée de Bataille. Elle est toute entière organisée autour d'une conviction tranchante : la volonté de durer et celle brûler ne sauraient faire bon ménage. La première signifie en effet servitude, subordination de l'existence en son présent à des fins à venir; durer égale peur de l'altérité, réduction à l'homogène, soumission à ces interdits qui délimitent le monde profane du travail. Durer implique limitation au possible, à l'orbe humain trop humain de ce qui est en notre pouvoir. Brûler signifie au contraire souveraineté, insubordination, brûler c'est porter l'existence à son point d'ébullition, vivre le présent comme une fête, s'insurger contre la fadeur blême d'une existence enlisée et sans air, 
affronter le tout autre ou l'hétérogène, transgresser les interdits, accéder au sacré et à la vérité de cri de l'Impossible...

4 Mais rien n'est plus retors aussi que la pensée de Bataille. Comment, en effet, la pensée pourrait-elle épuiser le fait d'être ${ }^{3}$, accéder jusqu'à l'horreur entrevue de la mort, elle qui est depuis toujours oubli de l'être en son ruissellement excessif, elle qui est dominée par une fièvre accumulatrice de sens et une volonté de réduction de l'être à l'homogène ? Faute d'être liée à une peur accablante, à l'horreur criée, la pensée me semble à côté du monde et de l'être qu'elle exprime: elle se dérobe ${ }^{4}$, selon moi, à la conscience d'une terrible situation ${ }^{5}$. Et plus la pensée est rigoureuse, écrit-il ailleurs, plus elle glisse sans garde-fous dans la nuit comme sur la pente d'un toit et dans un vent que rien n'apaise [...] répondant en un silence égaré [...] à l'appel de la mort ${ }^{6}$.

Cette pensée rouée nous met aussi au rouet en nous confrontant inévitablement à des contradictions insurmontables qu'on ne finirait pas de dénombrer. Ainsi si le projet remet le moment d'exister à plus tard, former le projet de sortir du projet nous enferme dans une aporie apparemment sans issue. Ou encore si le discours comme le travail ne sont jamais qu'une façon de conjurer la mort et une dénégation de la fuite affolée de l'être, comment écrire encore? Écrire ne pourrait alors signifier que s'engager à ne plus écrire de la sorte qu'à la fin ou on se tait, ou bien on trahit la souveraineté dont on parle ${ }^{7}$. Ou encore, ce qui revient au même, si penser est le contraire de dépenser ${ }^{8}$ alors l'adhésion à l'être ne peut pas vraiment advenir, l'expérience de la mort est impossible, cet instant de dissolution n'autorisant que le jugement disjonctif : ou la mort ou moi. Pour qu'elle soit possible il faudrait, vivant, en faire l'épreuve, se regarder cesser d'être ce qui est évidemment impossible. Ainsi on n'abolit pas le monde du projet et du possible, le monde du discours et de la production au bénéfice de la pure dépense. Le chemin trop court, le chemin tapageur du dionysisme sauvage ne mène nulle part et le Bataille de la maturité le sait bien : Nous pouvons évoquer furtivement les perspectives de l'éclat (mais) personne n'éclate: et nous continuons d'écrire, de publier, de lire ${ }^{9}$. Il est donc impossible de sortir de la littérature, impossible d'arracher le vêtement du langage : comme une tunique de Nessus il nous colle à la peau ${ }^{10}$, l'homme étant la même chose que le langage.

Rien de plus révélateur à cet égard que le travail et le livre du plus talentueux de ses commentateurs, Denis Hollier. Le travail d'édification des XII volumes des œuvres complètes à partir des papiers épars de l'œuvre dispersée d'un " anarchitecte » impose d'abord nécessairement une forme à l'inachèvement et donne le statut de livre à une "œuvre » exténuante qui, au nom de la violence du sacré, n'a cherché qu'à saper le système du savoir, qu'à se détruire et à se mettre en ruine. Par ailleurs, son livre sur Bataille, La Prise de la Concorde, est paradoxalement structuré par une riche et impeccable architectonique faite de rappels, de renvois, de rimes intérieures. Comment, dans ces conditions, prétendre rester fidèle à Bataille? Sa trahison, il l'avoue d'ailleurs dans la chute de ce livre si puissamment architecturé : Bataille est-il, ici, oublié, comme il le voulait. Ou trahi par l'insistance à maintenir ses traces? ${ }^{11}$

7 Cette difficulté à parler de Bataille nous a porté à mettre au cœur de notre propos le concept ludique de parodie; mais avant de caractériser la caricature dans laquelle on ne peut manquer de tomber quand il s'agit de parler de Bataille, il faut noter que ce concept peut jouer dans de multiples registres et d'abord dans le rapport ironique que Bataille entretient avec lui-même : je suis un saint, peut-être un fou, écrit-il par exemple, réécrivant sans doute un propos de Nietzsche ${ }^{12}$. Mais de même qu'un menteur ne peut 
pas dire qu'il ment, le saint ne peut se proclamer tel et le fou, abîmé dans l'absence d'œuvres, n'est fou que d'ignorer son état. On a donc bien dans cette affirmation comme un écho de cette parodie d'autobiographie, de ce livre fou qu'est Ecce homo, livre dans lequel Nietzsche mime et mine la croyance au «moi » en se livrant à un histrionisme qui bouleverse les canons de la représentation de soi.

Ce paradoxe de l'autoréférentialité ne fait que répéter celui d'une « œuvre » qui, disait Paulhan, refuse son statut rhétorique d'œuvre littéraire et verse dans le terrorisme en nous promettant de nous mener d'emblée au-delà tous les livres. Bataille ne fait pourtant ici que suivre ou reprendre l'exemple de Nietzsche dont il entend répéter l'expérience ${ }^{13}$. D'où une nouvelle occurrence où la parodie va jouer un rôle fondamental. Nietzsche lui-même en voulant être le parodiste de l'histoire universelle n'avait-il pas été "le premier» à faire de ce concept comme l'équivalent ironique de l'Aufhebung hégélienne ${ }^{14}$, donnant un sens nouveau aux mots de notre histoire au moment où, de cette histoire, il en réécrivait la tragédie? "Zarathoustra, écrit-il dans le Nachlass, adoptant une attitude constamment parodique envers les valeurs antérieures, par plénitude ${ }^{15}$. "Je suis chaque tous les noms de l'histoire ", écrira-t-il encore à Burckhardt, je suis, dans la ronde des dieux, à la fois Dionysos et le Crucifié. La parodie comme la relève (Aufhebung) hégélienne, détruit, annule, déconstruit mais en même temps conserve, remanie au marteau, dramatise, réévalue... avec la différence qu'à la suture du système Nietzsche et Bataille ${ }^{16}$ surtout - opposent le rien, le manque qui permettent un jeu à l'humour borgésien, un jeu essentiellement affirmatif ${ }^{17}$ qui vise dans le réel ce qu'il y a de plus réel, le manque, le trou, l'absence, pour finalement substituer sans fin à l'Origine défaillante la multiplicité des voiles et des interprétations. Tout le monde a conscience que la vie est parodique et qu'il manque une interprétation, écrit Bataille. Il manque l'interprétation qui viendrait arrêter le jeu ou la roue d'un monde qui n'a d'autre modèle que lui-même et interdire la circulation infinie des termes qui passent les uns dans les autres pour les mettre, avec ivresse, sens dessus dessous.

Pas de meilleur exemple sans doute de cette opération parodique de déconstruction créatrice qui se joue de la métaphysique sans jamais l'oublier que celle que Bataille fait subir au christianisme. "Nous voulons", écrit Bataille dans les Conférences sur le péché, "être les héritiers de la méditation et de la pénétration chrétienne [...] dépasser tout christianisme au moyen d'un hyperchristianisme et ne pas nous contenter de nous en défaire ". Bataille est celui qui, à partir d'une déconstruction du christianisme, a ouvert l'espace et l'époque athéologique qui sont les nôtres, réveillant de leur sommeil dogmatique, de leur incroyance satisfaite les libres penseurs échoués sur le terrain sec de l'athéisme: "Dieu n'est pas la limite de l'homme mais la limite de l'homme est divine... L'absence de Dieu est plus grande, est plus divine que Dieu $»^{18}$. Le nom de "Dieu» ne rend t-il pas paradoxalement fixe l'ouverture au dehors tout en le mettant a une distance infinie? Eckhard n'écrivait-il pas : «Prions Dieu d'être quitte de Dieu ? " ${ }^{19}$.

Mais si la notion méchante de parodie défait toute propriété, déstabilise toute identité à commencer par celle de l'auctor, elle est, comme le rire qui l'accompagne, toujours éclatée et multiple car elle se dit en plusieurs sens; bien malin qui voudrait en dénombrer tous les états... Parodia est un mot grec formé du préfixe para qui veut dire à côté et de la locution nominale odè, qui signifie poésie et chant ${ }^{20}$. Il désigne au XVII ${ }^{\mathrm{e}}$ siècle où le mot apparaît l'imitation burlesque d'une œuvre sérieuse et par extension se dit d'une œuvre détournée à des fins plaisantes. Au sens figuré il renvoie au siècle suivant à une contrefaçon ou une caricature qui ne reproduit que quelques apparences. 
On pourrait en distinguer plusieurs espèces ou plusieurs variétés suivant que l'on augmente ou que l'on diminue la distance indiquée par le préfixe para, distance qui donne son rang à un rire qui chaque fois témoigne du degré de la prise de distance. Quand la distance est minimale elle peut être inconsciente et n'apparaître qu'à un tiers. Quand la transposition est pleinement consciente, son sens varie suivant l'intention de l'auteur : elle peut être ludique, satirique ou sérieuse même si dans ce dernier cas il n'existe pas vraiment de mot pour désigner cette réécriture intertextuelle qui est la marque propre de la littérarité ${ }^{21}$.

11 Mais il est clair que dans le cas qui nous occupe ces distinctions se trouvent quelque peu brouillées dans la mesure où l'opposition jeu/sérieux perd de sa pertinence (le jeu chez Nietzsche est la marque du " grand sérieux»), dans la mesure aussi où la parodie, le retour ou le contre-chant va prendre chez Bataille et Nietzsche, chez Bataille répétant l'expérience de Nietzsche, une envergure ou une importance "capitale»: le retour c'est l'éternel retour qui fut d'abord une catégorie musicale désignant un motive, un leitmotive qui revient et recommence toujours sans avoir jamais commencé ${ }^{22}$. Le retour c'est l'éternel retour des mots, des pensées, des citations, des expressions, des anecdotes de sorte que chaque texte peut être considéré comme une sorte de palimpseste dans lequel transparait les traces de l'écriture préalable; toute littérature ne commence-t-elle pas par la pratique originelle de la réécriture ${ }^{23}$ ? La parole de Nietzsche mise ici en exergue apparaît en ce sens comme un miroir interne, un récit spéculaire, une mise en abyme réflexive de cet interminable procès, une illustration de cet étrange oxymore : originaire réécriture ${ }^{24}$.

Tentons d'entrer dans la ronde de ces contrepoints étourdissants en déterminant, en ce qui nous concerne, les sens et les occurrences de la parodie.

Le modèle de la parodie, volontaire, intentionnelle et à visée destructrice, de la parodie qui cherche à inverser la perspective d'un texte premier, à en retourner le sens et à en prendre le contre-pied nous est donné avec le Zarathoustra de Nietzsche ${ }^{25}$. La nature parodique du Zarathoustra est inscrite jusque dans le nom de son héraut éponyme puisque le réformateur religieux, le prophète persan du Bien et du Mal, est censé reconnaître son erreur pour devenir le protagoniste d'une œuvre qui annonce la mort de Dieu, l'éternel retour et la transmutation de toutes les valeurs. La parodie des textes sacrés et philosophiques est omniprésente dans le Zarathoustra: dès le Prologue sont parodiés aussi bien le soleil platonicien que la figure du Christ. La christologie paulinienne de la kénose est la basse continue de tout le poème scandé par le thème mystique de la surabondance et du retrait: «j'aime celui qui donne, j'aime celui qui sombre et ne cherche pas à se conserver... » Mais c'est dans le chapitre intitulé La Fête de l'âne, que culmine sans doute la parodie et que sont affirmées son ambigüité et les conditions de sa signification subversive. La Fête de l'âne, est une parodie à la seconde puissance, une parodie de la parodia sacra censée commémorer la fuite en Égypte, une parodie du rituel carnavalesque si bien analysé par Bakhtine. Car, comme on le sait, un tel rituel finit toujours par restaurer l'ordre qu'il singe, par renforcer la hiérarchie qu'il renverse, par rétablir les valeurs qu'il moque si bien que Zarathoustra est tout à fait fondé à reprocher aux hommes supérieurs (l'expression est empruntée à Paul Bourget) d'avoir mis en scène cette fête parodique ${ }^{26}$.

14 Mais pour être une œuvre parodique, le Zarathoustra n'en est pas moins, pour Nietzsche, l'œuvre la plus sérieuse qui soit et c'est ici que Bataille va intervenir. Il est en effet des cas de parodie involontaire où la parodie inconsciente d'elle même 
n'apparaît comme telle qu'à un tiers. On a alors à faire à une parodie cachée et honteuse, à une contrefaçon caricaturale. C'est par exemple le cas du kitsch qui se développe à l'ère de la reproduction technique lorsque la bourgeoisie n'a plus de projet historique et se contente de recopier, souvent à moindre frais, la splendeur des œuvres du passé : les châteaux de Louis II de Bavière relèvent, par exemple, de cette catégorie mais, d'une certaine façon aussi, toute l'œuvre de Wagner. Or c'est en proie à une rivalité mimétique avec Wagner, au moment où ce vieil enchanteur meurt à Venise, que Nietzsche va lui opposer son Zarathoustra; mais ce Zarathoustra n'est-il pas à sa manière aussi un autre grand guignol ? Ce nouveau messie théatral n'est-il pas, ainsi que le disait Thomas Mann, "un fantôme vacillant au bord du ridicule", une parodie involontaire des grandes figures, de la grande quincaillerie de la tétralogie?

C'est bien sur ce mode que Bataille va se rire de Nietzsche et stigmatiser la pulsion ascensionnelle, l'échappatoire et la maladie icarienne, c'est-à-dire, tout simplement le péché d'idéalisme que trahit que révèle le "sur» de ce surhomme (comme dans le «sur» de surréalisme) dont Zarathoustra est le héraut ${ }^{27}$. La volonté d'inverser la perspective, de renverser sur le mode parodique le très haut en très bas va conduire Bataille à opposer au préfixe « sur ", le « a » privatif d'Edwarda, d'acéphale, d'Olympia... de telle sorte que toute son œuvre a pu être comprise comme une entreprise forcenée de désublimation.

16 La question s'était déjà posée avec acuité à propos du statut de l'œuvre de Sade et de son appartenance à la littérature ${ }^{28}$. La revendication bataillienne de la valeur d'usage de Sade est expressément dirigée contre les surréalistes accusés d'avoir textualisé son œuvre jusqu'à la faire entrer à la bourse des valeurs littéraires. «À louer Sade on édulcore sa pensée ", écrit-il dans la préface à la deuxième Justine. Bataille voulait plutôt, comme Debord, hurler avec Sade et voir son influence se traduire concrètement par l'insurrection des parties basses de l'individu comme de la société ${ }^{29}$. Il y avait donc bien chez Bataille un parti pris résolument sadien de désublimation, une volonté de parler bassement de ce qui est bas, une volonté scandaleuse d'aller toujours plus bas et d'aimer la pourriture jusqu'à la honte. Fidèle à Sade, fidèle à celui qui porte la mauvaise nouvelle de l'accord des vivants avec ce qui les tue, Bataille a cherché, dit fortement Surya, à ce que rien n'échappe à la mort, à faire que la mort soit sans reste, c'est à dire qu'elle soit enfin sans recours et sans issue ${ }^{30}$. «L'écriture est impuissante: il me manque le visage et la nudité d'une prostituée pour dire assez bas de la vie humaine qu'elle a fait d'elle-même une façade et que la débauche la rend à la vérité ». Seule une hilarité bestiale qui nous ferait pousser des cris de porcs pourrait, semble-t-il, disloquer la langue, ébranler la sécurité du décor dressé par le savoir et le travail et laisser entendre la voix barbare et fêlée qui vient du sexe.

17 On peut pourtant s'interroger sur cette désublimation que, d'une façon quelque peu outrancière, on monte en épingle. Ne serait-elle pas autre chose qu'une imposture ou qu'une escroquerie? La littérature -et plus généralement l'art- ne sont-ils pas nécessairement une métaphore, condamnés à rester toujours enfermés dans un monde de signes de sorte qu'il ne peut y avoir, par principe, d'art désublimé ${ }^{11}$ ? Comme le dit Roland Barthes à propos de Sade, "écrite, la merde ne sent pas; Sade peut en inonder ses partenaires, nous n'en recevons aucune effluve, seul le signe abstrait d'un désagrément ${ }^{32}$ ». Et quand Sade, avant que la merveilleuse corolle ne pourrisse impudiquement au soleil, effeuillait ses roses sur le purin de la fosse, comment éviter que la perte ici ne se constitue en cliché et ne renaisse comme fleur dans le jardin des lettres ${ }^{33}$ ? Un art qui se 
prétend désublimé ne serait-il pas nécessairement un art mensonger, mensonger parce que justement, comme le disait Baudelaire ${ }^{34}$, il aurait négligé de mentir? Le paradoxe n'est-il pas là ? C'est lorsque l'art s'affirme comme tel, lorsqu'il s'affirme comme faux, comme une erreur ou une imposture voulue, comme un mode spécifique de fiction et d'illusion qu'il peut être infiniment plus près du vrai...

Serait-ce donc maintenant à Bataille qu'il faudrait objecter, comme à un parodiste involontaire, la fausseté de son chant? L'assis de bibliothèque, le nouveau mystique ne serait-il qu'un imposteur, qu'un terroriste, qu'un misologue sacrifiant auteur et œuvre, condamnant écriture et rhétorique ${ }^{35}$, pour promettre feu et flamme, horreur et jouissance, convulsion et consumation à notre vie d'encre trop souvent vouée à un assouvissement ludique, futile et illusoire? "Ceci» ne serait donc pas un livre? L'écriture serait-elle uniquement destinée à se briser pour ne plus être que le cri de l'émotion ${ }^{36}$ ?

19 Et pourtant Bataille, dès L'Expérience intérieure où il parle de l'ultime plaisanterie de son expérience ${ }^{37}$ et dès Histoire de rats, livre dans lequel la réécriture de Proust va devenir aussi de plus en plus insistante, a implicitement répondu: on ne peut opposer la terreur et les lettres, la littérature est comme la salamandre qui se nourrit de flamme et la terreur sacrificielle ne peut exister et s'accomplir que par la médiation du simulacre littéraire ${ }^{38}$, Incipit coemedia ! Incipit parodia !

"J'écris couché, à trois heures, il pleut à verse; il me faudrait m'en aller nu, sous la pluie, un bandeau sous les yeux, mourir en mangeant de la terre ». Comment exprimer plus lyriquement et rendre mieux communicable ce mouvement sans fin de déchéance et de chute, cette obsession de la destruction qui fascine Bataille? Mais tout cela opère dans un discours, comme le souligne ici le conditionnel. L'ambition désublimatrice (selon l'expression de Denis Hollier) demeure mais elle ne peut être qu'une ambition et rien ne peut faire que le statut symbolique de l'œuvre ne la relève. L'art est donc bien un mensonge, ceci est donc bien un livre et personne ne peut dire le contraire. S'agit-il de n'en faire qu'un livre, de n'en faire qu'une œuvre ? Cette proposition non plus personne ne peut la soutenir, car si l'art n'est pas la vie, si l'art est bien un mensonge en rupture avec le réel, c'est aussi un mensonge qui dit la vérité. Que nous importerait un livre qui ne nous mènerait pas au-delà de tous les livres ? Et, comme l'écrivait Bataille dans la préface du Bleu du ciel, comment nous attarder à des livres auxquels, sensiblement, l'auteur n'a pas été contraint ${ }^{39}$ ?

21 Dans la mesure où ils le peuvent [...] écrit Bataille, les hommes recherchent les plus grandes pertes et les plus grands dangers. La séduction extrême, écrit-il aussi, est peutêtre à la limite de l'horreur. On pense au Léontios de la République de Platon ${ }^{40}$ : après s'être couvert le visage et avoir lutté contre lui-même, il finit par céder au désir (épithumia), par ouvrir tout grands les yeux et à courir vers les cadavres... Mais comment regarder la mort en face, comment maintenir ce qu'il y a de plus terrible, comment maintenir l'œuvre de la mort, pour reprendre les mots de Hegel, sans passer par le medium littéraire où artistique? Le subterfuge, le simulacre, la comédie du spectacle ou de la représentation ${ }^{41}$ sont nécessaires pour que l'impossible puisse devenir possible et Bataille ne cesse de retrouver ainsi à sa manière le fond orphique du geste littéraire, la topique immémoriale du supplice et de son échec ${ }^{42}$ tout en renouant avec la plus classique des théories, celle de la mimésis, celle de la Poétique d'Aristote. La putréfaction cadavérique exemple superlatif de l'informe et de l'ignoble ne peut être supportée que grâce au trans-port de la métaphore (méta-phoros) et à l'épuration 
artistique ${ }^{43}$. Prenant l'exemple de la littérature la plus populaire, la littérature policière qui, plus qu'une autre fait toujours fond sur la représentation du danger et de l'horreur, Bataille montre que, grâce à elle, le lecteur peut jouir par procuration du sentiment d'être en danger que nous donne l'aventure d'un autre ${ }^{44}$. Si l'horreur est le fond des choses, alors l'art, exercice de la cruauté est là pour contenir l'horreur. En tous les sens du terme, il la refreine mais il la garde en réserve aussi et s'y alimente nous permettant ainsi de ne rien éluder et de vivre à hauteur de mort.

Ce n'est pas par un coup de pistolet que l'on entre dans l'absolu, écrivait Hegel et ce n'est pas par enchantement qu'on abolit le monde du projet et du possible, le monde du discours et du travail au bénéfice d'un plain chant pulsionnel écrit sous la dictée de l'inconscient, d'une écriture antirhétorique et sacrificielle, au profit du don et de la pure dépense... En revanche, dans ce monde humain trop humain, il est possible de se remettre au discours qui règle tout mais sur le mode dramatique, de dramatiser donc en réécrivant, par exemple, les exercices spirituels d'Ignace de Loyola, de mimer verbalement des images bouleversantes ${ }^{45}$. Autant de façons de parodier, de trouver des points de rupture, des expériences limites et transgressives qui trouent le discours et le rendent poreux et transparent au tout autre ${ }^{46}$. C'est dans le medium littéraire, dans la fiction marqués par une écriture, par des techniques, par la récurrence de thèmes et d'enchaînements narratifs, de procédés rhétoriques immédiatement reconnaissables ${ }^{47}$ que peut se défaire un peu l'ordre où nous étouffons. Ce sont les parois de la maison ou de la prison du langage que le style de Bataille a patiemment laminés, jusqu'à ce qu'il laisse deviner, furtivement, l'image du dehors : que serions-nous sans le langage? Seul, il révèle, à la limite, le moment souverain où il n'a plus cours ${ }^{48}$.

On ne sort donc pas du jeu, on ne va pas au-delà du sérieux ou, comme le dit Bataille, le sérieux de la mort et de la douleur reste toujours la servilité de la pensée. « Aucune page de cette vie et la dernière moins qu'une autre ne saurait être prise au sérieux» et "si nous nous démenons comme des satyres c'est pour traiter de façon parodique cette vie qui ne cesse de nous regarder de façon si grave et si pathétique, cothurnes aux pieds ${ }^{49}$ ». Incipit tragoedia? Incipit parodia ${ }^{50}$

24 Parmi toutes les interprétations de Nietzsche, celle de Bataille est la seule à avoir pris acte de cette situation nouvelle : non seulement toute position de surplomb à l'égard d'une pensée essentiellement incorporée est devenue impossible, mais la notion de parodie en devenant le proprium et l'ipsissimum de l'homme moderne entame profondément le grand habitus culturel de la philosophie occidentale pour lequel tout procédait ou tout convergeait dans l'Un, tout se rassemblait dans la ténébreuse et profonde unité. Désormais l'Un se divise en deux et la répétition devient originaire : que l'on admire, que l'on décrie, que l'on explique, que l'on joue, que l'on cherche à parfaire... on ne cesse de répéter - et on répète dans la différence - ce qui, de soi-même, déjà s'était répété, ce qui originairement résonnait. En ce sens le maitre livre de Deleuze (Différence et répétition,1968) et l'article de Derrida (La Différance) inaugurent la pensée moderne comme pensée originairement dia-bolique de la différence ou, ce qui revient au même, dans le deuil de l'origine et de la pure et pleine présence à soi, comme pensée de la répétition de ce qui n'a lieu qu'en se différenciant ${ }^{51}$.

N'ayant nous même plus rien à dire et prenant plaisir à répéter, nous sommes entrés dans le jeu de la parodie ${ }^{52}$ et nous avons voulu accompagner de notre main gauche, cette fugue ou ce contre-point sans préjuger de savoir si, de ce travail épigonal, de cet interminable travail de "collage», du nouveau, de nouveau, pourrait advenir ${ }^{53}$. Mais 
portant et répétant la somme énorme d'une histoire qui est notre histoire ${ }^{54}$, loin de la mélodie postmoderne et de ses amères "rengaines", indifférents au travail d'exhumation archéologique et de ses soucis antiquaires, nous avons été gagnés par la surprenante capacité de résurgence de ces œuvres et nous voici prêts, désormais, à saluer l'aurore sa promesse et sa chance.

\section{NOTES}

1. Jusqu'à notre lecture de Montaigne, Du repentir, Actes-Sud, 2001.

2. . Cité par Denis hollier, La Prise de la Concorde, Paris, Gallimard, 1974, p. 57.

3. . «L'expérience intérieure », OC V, 16.

4. . Penser, ce sera toujours, selon la formule emblématique et le paradigme érotique de L'Expérience intérieure, penser comme une fille enlève sa robe, penser comme une fille qui s'expose aux jeux vertigineux de l'amour : sa beauté ne s'enlève jamais que sur le fond déchiré qui la menace, sur le vide mortel que sa robe dissimule et trahit : il n'y a jamais de beauté sans horreur et c'est à la nuit que le jour doit son éclat. C'est le leitmotive de notre livre sur L'Art (Ellipses, rééd. 2011).

5. . OC VI, 367.

6. . OC XII, 278. Thanatophilie? Mais le monde souverain n'a une odeur de mort que pour l'homme subordonné ; c'est au contraire le monde servile de la pratique, celui de la mort refoulée et rentrée, qui sue l'affolement et l'angoisse.

7. . OC XII, 127.

8. Donner, sacrifier, perdre ou consumer sans réserve - au sens bancaire et en tous les sens du terme - ne peuvent procéder de la décision d'un sujet libre. Dans ce cas, en effet, il se réapproprierait son don, ce qui le priverait, dès lors, de toute gratuité et générosité. Comme le dit Blanchot le don de souveraineté n'est encore que titre de souveraineté, enrichissement de gloire et de prestige, fût-ce dans le don hérö̈que de la vie. L'Essai sur le don de Marcel Mauss qui inscrit dans une logique de la dette et de la solidarité (celle que l'échange monétaire va éteindre, pagar, payer, c'est apagar, éteindre) ne subvertit en rien la loi d'airain de l'économie, la balance irréfutable, le livre de compte qui exigent que tout don soit compensé par un contre don. Bataille qui veut renverser l'économie, penser l'économie à la mesure de l'univers, s'épuise à penser le don ou la dépense par-delà le principe d'utilité, comme une réponse à la donation, au il y a qui se dit en Allemand, Es gibt. Ilse mesure là encore à une figure de l'impossible. Voir notre article : «Écologie Bataillienne? ", in Il Particolare, 2012, à paraître.

9. . OC XI, 55.

10. . L'autre occurrence de ce mythe concerne Nietzsche, justement. « Vis-à-vis de (Nietzsche) je brûle, comme par une tunique de Nessus, d'une anxieuse fidélité... C'est d'un sentiment de communauté me liant à Nietzsche que naît en moi le désir de communauté, non d'une originalité isolée ", OCV 39. Déjanire, pour s'assurer de sa fidélité, avait fait passer à Héraclès ce vêtement empoisonné qu'il ne put enlever sans arracher en même temps des lambeaux de chair. Cela conduisit le héros à s'immoler par le feu et à mourir brûlé vif de douleur. Un congé définitif est ainsi de nouveau signifié à une innovation qui se voudrait radicale.

11. . Ibid., p. 296.

12. . «Ne me prenez pas pour un saint plutôt pour un satyre!» écrit-il dans ce livre au titre parodique, Ecce Homo. 
13. . L'expérience chez Bataille n'est ni l'Erlebniss (le vécu, trop psychologique) ni l'Erfahrung hégélienne accumulée au cours du temps, c'est le fait, pour un sujet arraché à lui-même de s'exposer au péril (periculum) jusqu'aux limites de l'invivable et de l'impossible. De cela, la science qui se veut sans sujet ne veut rien savoir.

14. . La répétition bataillienne du système hégélien magistralement analysée par Derrida dans «Un hégélianisme sans réserve » (L'Écriture et la Différence, Seuil, 1968) est le meilleur exemple de cette Aufhebung parodique. "Absurde ruée de l'ipse voulant être le tout», écrit Bataille dans L'Expérience intérieure, visant le désir de totalité et de maîtrise qui masque la vérité de la castration, de la déchirure sexuelle, de la section ou de la sexuation. L'érotisme pensé à partir du paradigme du sacrifice fait définitivement échec à toute tentative de totalisation et de maîtrise philosophique et redonne tout son sens à l'existence de chacun : "s'il y seulement de l'univers inachevé, chaque partie n'a pas moins de sens que l'ensemble ».

15. . Euvres philosophiques complètes, XII, 1885-1887, 7 (54), p. 303. Gallimard.

16. . Voir Bataille Anus solaire et Nietzsche Historia in nuce: La plus sérieuse parodie, (die ernsthäftigste Parodie) que j'aie jamais entendu est celle-ci : "Au commencement était le nonsens, et le non-sens était, par Dieu! et Dieu, divinement était le non-sens ». Opinions et sentences mêlées, § 22. Cf. Lettre à Carl Fuchs, juin 1878.

17. . Gai Savoir, § 382.

18. OC V, 350, OC XI, 236. OC VI, 315. C'est ce mouvement profond de subversion de l'intérieur, de déconstruction que nous avons voulu retrouver, que nous avons voulu répéter dans «Le christianisme en héritage » (Le Portique, 2011) en tentant, à notre manière, de retourner la vaste terre du sacré et de libérer, pour la pensée, la possibilité de se mesurer à l'incommensurable.

19. . Cité par NIETZSCHE, Le Gai Savoir, § 292.

20. . " Ôdè c'est le chant ; para : "le long de", “à côté” : parôdein d'où parodia, ce serait “donc ?", le fait de chanter à côté donc de chanter faux, ou dans une autre voix, en contre chant - en contrepoint -, ou encore de chanter dans un autre ton: déformer donc ou transposer une mélodie ", G. GENETTE, Palimpsestes, Paris, Seuil, 1982, p. 20.

21. Il y a littéralité lorsque les livres renvoient à d'autres livres beaucoup plus qu'à un référent extra-textuel. Cette intraréférentialité est selon Jackobson le marqueur de la fonction poétique.

22. . Ph. LACOUE-LABARTHE, Typographie I.

23. . Tout mot, depuis que le monde existe, est un écho de mémoire. Chacun de nous copie sur l'autre, imite, pastiche, emprunte, s'inspire, vole ou plagie (même si la notion de plagiat est solidaire d'une définition très récente de la propriété intellectuelle car auparavant tout, les mots et les idées, était à tout le monde) et avance en claudiquant, avec toujours un regard en arrière. En ce sens, écrivait Giraudoux, «le plagiat est la base de toutes les littératures, excepté de la première, qui d'ailleurs est inconnue ».Térence l'affirmait déjà, plus d'un siècle et demi avant notre ère : « Rien n'est dit qui n'ait été dit ». Bakhtine dans sa Théorie de la littérature, éditions du Seuil, 1965, p. 50 écrivait: "Notre pensée ne rencontre que des mots déjà occupés, et tout mot, de son propre contexte, provient d'un autre énoncé déjà marqué par l'interprétation d'autrui ». Dans ses Propos sur l'éducation, Alain disait de même : «Il n'y a qu'une méthode pour inventer, qui est d'imiter. Il n'y a qu'une méthode pour bien penser, qui est de continuer quelque pensée ancienne et éprouvée ».

24. . Siamo contenti? "Nous sommes contents? Je suis dieu, j'ai fait cette caricature». Si la parodie est bien, comme le dit G. Genette, une «transformation textuelle à fonction ludique " alors en effet l'attitude de parodiste que Nietzsche ou Zarathoustra adopte à l'égard de toutes les valeurs antérieures est bien un jeu, un rire, une conséquence de sa propre richesse ou de sa propre abondance. Mais, dans cette dernière lettre à Burckhardt, pourquoi ce mélange de grandiloquence et de burlesque, d'orgueil et d'humilité, pourquoi ici ce double travestissement ? Pourquoi dire le contentement, la liesse ou la gaieté dans une langue étrangère, celle des troubadours, celle du Gai Saber ou de la gaya scienza ? Et pourquoi cette identification à Dieu, au Prince, au princeps s'accompagne-t-elle de la revendication de la caricature ou de la parodie qui, 
par définition est toujours seconde et dérivée ? Pourquoi est-ce au moment où Nietzsche passe de l'autre côté, bascule définitivement dans la folie que la maladie lui donne l'illumination?

25. Sur cet art d'inverser les perspectives et sur le rapport à Zoroastre, voir Ecce Homo.

26. . Voir Paolo D’IORO, «Genèse, parodie et modernité dans Ainsi parlait Zarathoustra ", in Lecture d'une cuvre, Also sprach Zarathustra, Paris, Éditions du Temps, 2000. Sur l'âne, sur l'ambivalence de cette figure sacrilège du Dieu chrétien, sur son infatigable Ia (ita est) donné à ce simulacre de doctrine qu'est le retour de l'identique, voir Pierre KLossowsKI, « Nietzsche, le polythéisme et la parodie », in Un si funeste désir, Paris, Gallimard, 1963, p. 227.

27. Mais Nietzsche dans la préface du Gai Savoir cité plus loin (note 46) s'était lui-même parodié en opposant un incipit parodia à l'incipit tragoedia de l'aphorisme 342 (qui reprend le prologue du Zarathoustra), formule qui lui même parodiait l'incipit comoedia de la Divine Comédie de Dante...

28. . Voir notre « Bataille avec Sade ", in Il particolare 11.

29. . Voir notre article « G. Bataille et le retrait du politique » in L'Enseignement philosophique, $53^{\mathrm{e}}$ année, $\mathrm{n}^{\circ}$ 3, Février 2003.

30. . M. SURYA, «L'imprécation littéraire », Matériologie I, Farrago, 1999.

31. Sublimation le mot appartient d'abord au contexte alchimique. Nietzsche l'utilise métaphoriquement lorsqu'il cherche a effectuer la transmutation en or de ce qui fut le plus haï et méprisé par l'humanité. Mais n'y t-il pas bien des textes où Bataille lui aussi, à son corps défendant peut-être, utilise le lexique de la sublimation? À commencer par le mot gloire, hyperbole paradigmatique du sublime.

32. . Sade, Fourier, Loyola, Seuil, 1970, p. 179.

33. Jean PAULHAN, Les Fleurs de Tarbes, Paris, Gallimard poche, 1990. Élisabeth ARNOULD-BLOOMFIELD, Georges Bataille, la terreur et les lettres, Perspectives, Septentrion, Presses Universitaires, 2009. Voir aussi G. BATAILLE, « Le Langage des fleurs », OC I.

34. . Ch. BAUdelaire, Critique d'art, Paris, Pléiade II, 1985, p. 668.

35. Voir la célèbre définition de la poésie : le sacrifice où les mots sont victimes, (ce sont des mots qui se brûlent écrivait aussi Léon-Pol Fargue). Ce privilège du sacrifice, de l'extase et du supplice sur le langage est un leitmotive de L'Expérience intérieure où la rhétorique est considérée comme sans importance dans la moisson de son livre OC V, 178).

36. . « René Char et la force de la poésie », OC XII, 127.

37. OC V, 181.

38. OC $\mathrm{V}, 181$.

39. . OC III, 281.

40. . République, $439^{\mathrm{e}}-440 \mathrm{a}$.

41. . «Hegel, la mort, le sacrifice», OC XII, 326 s. «Dans le sacrifice, le sacrifiant s'identifie à l'animal frappé de mort. Ainsi meurt-il en se voyant mourir, et même en quelque sorte, par sa propre volonté, de cœur avec l'arme du sacrifice. Mais c'est une comédie ».

42. . Élisabeth ARNOULD-BLOOMFIELD, op. cit. Par exemple : Par delà l'expérience il est nécessaire, à cette fin, de s'en remettre à la fiction » (OC VIII, 551) ou encore Bataille évoque la "nécessité d'aller au moins par la pensée jusqu'au bout de la séduction » (OC VIII, 149).

43. . Poétique, IV, $48 \mathrm{~b} 12$.

44. . « L'érotisme », OC VII.

45. . «Si nous ne savions dramatiser, nous ne saurions sortir de nous-mêmes ». «L'expérience intérieure », OC V, 23, 26, 139.

46. . Mutatis mutandis c'est ce que nous avons tenté de faire en utilisant le concept bataillien d'altération dans notre ouvrage sur les arts premiers. Voir La Passion de l'origine, Ellipses, 2006.

47. Techniques analysées dans un article de Catherine CUSSET intitulé: «Techniques de l'impossible » in Bataille après tout, Paris, Belin, 1995.

48. . Voir L'Érotisme, conclusion. 
49. . Lettre de Nietzsche à Sophie Ritschl, 2 juillet 1868.

50. . Voir Le Gai Savoir, Préface. « Mais celui qui le pourrait me pardonnerait à coup sûr bien plus qu'un peu de folie, d'exubérance, de "gai savoir", - par exemple la poignée de chants qui sont désormais ajoutés à ce livre (ceux d'après lesquels nous avons extrait le petit poème sur SilsMaria que nous avons cité en exergue) - chants dans lesquels un poète tourne tous les poètes en ridicule de manière difficilement pardonnable. - Ah! Ce n'est pas seulement sur les poètes et leurs beaux "sentiments lyriques" que ce ressuscité doit passer sa méchanceté : qui sait quel genre de victime il recherche, quelle monstrueuse matière à parodie le charmera sous peu? "Incipit tragoedia" lit-on à la fin de ce livre dangereusement inoffensif : qu'on se tienne sur ses gardes! Quelque chose de prodigieusement mauvais et méchant s'annonce : incipit parodia, à n'en pas douter... »

51. . Sur ce thème, voir J.-L. NANCY, Variations sur la reprise, Conservatoire Strasbourg éditions, 2010. La présence du présent, disait déjà Augustin, n'est qu'en cessant d'être, immédiatement dédoublée et séparée de soi. Sur le jeu de l'un qui se divise et se défait, voir aussi Dans quels mondes vivons-nous avec Aurélien BARRAU, Galilée, 2012.

52. . On voit qu'en ce sens la parodie n'est pas simplement l'instrument provisoire d'une liquidation qui ménagerait le parodiste, lui permettrait de s'épargner et de se mettre hors jeu : elle est plutôt, en son sens majeur, une déconstruction créatrice dans laquelle le parodiste, n'étant plus maître du jeu, est pris, quitte à entrer avec humour dans cette ronde ou cette spirale sans fin ici mise en scène.

53. Ce travail devenu travail de prospection et non d'exhumation des textes ne fait qu'un avec l'ouverture et la fuite en avant du sens.

54. . Gai Savoir, § 337.

\section{RÉSUMÉS}

Comment écrire sur Bataille sans s'épargner et se mettre soi-même hors jeu sinon en entrant dans le jeu de ce qu'on appellera, en un sens majeur, la parodie ? Dans ce retour, dans cet éternel retour des pensées et des mots - dans cette déconstruction créatrice qu'est la réécriture Bataille n'y entra-t-il pas lui même en écrivant sur Nietzsche?

How can one write about Bataille - sparing oneself by putting oneself beyond reach - if not by stepping into what can only be called, strictly speaking, parody? Didn't Bataille himself enter this process, this "eternal return" of words and thought - this creative deconstruction called rewriting - when he chose to write about Nietzsche?

Wie kann man ohne sich zu schonen und ohne sich selbst ausser Spiel zu bringen über Bataille schreiben? Vielleicht im Spiel der Parodie? Hat Bataille nicht selbst auf diese Art über Nietsche geschrieben? 